\title{
Interoperability of Standards to Support Application Integration
}

\author{
Em delaHostria \\ Rockwell Automation, USA, em.delahostria@ra.rockwell.com
}

\begin{abstract}
One of the key challenges in the design, implementation, operation, and maintenance of an industrial automation system is the selection of appropriate standards to facilitate system integration. A significant aspect of the integration challenge is determining whether the standards-conformant components making up the system will operate with each other. The interoperability of these components depends on which interfaces are used in their implementation.
\end{abstract}

This paper addresses how such interoperability issues can be addressed, early in the system requirements-capture and design stages and throughout its lifecycle, by an application integration framework. The framework provides an approach to identify (a) the necessary integration model views of a control system application and (b) the applicable standard interfaces within these model views. Given the set of required interfaces and the set of selected options for each interface, automation system suppliers can assess design and implementation choices that meet the application's requirements.

\section{SITUATION}

In deploying an industrial automation system that meets the requirements of an application, the necessary functions may be enabled either as designedin functions or as add-on functions. The cost of enabling any function, during a production activity, is clearly dependent on the flexibility of the underlying control architecture used and the resources employed to realize the automation control system. The choices of interfaces, made at design time, for the resources that implement the functions determine how well these functions can be performed in a coordinated manner at run time. 
In selecting these resources, it is important to insure that the resource interfaces provided are not only compatible to realize system integration but also interoperable to achieve application interoperability. This situation can be difficult to realize especially if the resources are supplied from multiple vendors. One approach to realizing a high level of integration and a fuller degree of interoperability is to select interfaces that conform to international and industry standards. However, it is also equally important to verify that that the standards specifying the interfaces are compatible and interoperable, given the requirements of a manufacturing application. This aspect is key to the practical use of an application integration framework.

\subsection{Model view of a manufacturing enterprise}

In this paper, the model view of a manufacturing enterprise consists of an organization whose main activities are partitioned according to the enterprise functions described in the IEC 62264, (2002) draft standard. The key areas of interest cover the activities in the design, development, operation, and maintenance phases of a manufacturing application process lifecycle. These activities are very interdependent with similar activities in the design, development, production, and distribution phases of a product lifecycle. In most cases, the production phase of a product's lifecycle overlaps with the operation and the maintenance phases of a manufacturing application's process lifecycle. However, the resources employed in the process can be considered to have their own lifecycle, since some of these resources are consumed while others break down and then either repaired or replaced.

This particular but partial model view is derived using a methodology specified in ISO 15704, (2001), an international standard defining a generalized reference architecture of a generic enterprise. In this standard, the activities performed during an enterprise's operational phase usually include all those activities associated with the lifecycle of a product. In some cases, some of these product lifecycle activities, such as product distribution and field support are each accomplished by a different enterprise in the supply chain.

In practice, different standards are used to describe the lifecycle-specific activities. For instance, a description of an integrated product and manufacturing process planning activity could be realized if the product data, expressed in conformance with the ISO 10303, (1999) standards, can be used to drive the design, development and operation of a manufacturing process, expressed in a standard specification language like ISO 18629, (2002). The integrated description can be generated if the related definitions used in product data exchanges (ISO 10303, 1999), in production process sequences (ISO 18629, 2002), and in the production information exchanges (ISO 
$62264,2002)$ were harmonized to facilitate the construction of an integrated product and process model view.

\subsection{Model views of an enterprise's manufacturing system}

During the operating phase of a manufacturing process lifecycle, a required function can be performed if it is already enabled or it may be installed, configured, and executed on demand. A key condition to be satisfied is that interfaces of the resources used to perform the function are configured to work with the corresponding resource interfaces of the other functions involved in a target manufacturing application. These conditions can be illustrated using various model views of a target application. The manufacturing application model view provided by ISO 15745, (2002) relates the process to the products produced and to the manufacturing resources utilized.

The application integration framework defines the elements and rules for composing this model view of a manufacturing application. A typical model view of an integrated manufacturing application consists of one or more processes that are enabled by a group of resources of various types - machinery, devices, personnel, software, materials, utilities, and other forms of equipment. The information exchanged between the resources can also be modeled as one or more software units that include various types - data items and structures, databases, communication protocols, and other software components that handle the data items. In the ISO 15745, (2002) standard, the specific interfaces between the resources are enumerated and their compatible options are summarized in a concise statement of interoperability, expressed in terms of XML statements and associated schema (XML, 2000/2001).

\section{FRAMEWORK FOR INTEGRATION AND INTEROPERABILITY}

To understand how the processes in an application cooperate with each other, a set of interfaces are assumed to be configured to enable the flow of materials, information, or any other resource needed to accomplish the requirements of an application. An example of such an application is a material handling application where the objective is to move raw materials, inprocess goods, and finished goods according to the needs of an enterprise's manufacturing and business processes. 


\subsection{Framework elements and rules}

In ISO 15745, (2002), a manufacturing application is modeled as a set of manufacturing processes, resources and information exchanges for the purpose of expressing the integration requirements. Integration models are expressed in terms of the Unified Modeling Language (ISO/IEC19501, 2001) conventions and are used to identify the required interfaces and to show how these choices meet the functional and performance requirements of the application (see Fig. 1 below).

In terms of UML class diagrams, an integration model of a Manufacturing Application class consists of a set of Manufacturing Process classes, a set of Manufacturing Resource classes, and a set of Manufacturing Information Exchange classes. Further, a generic Manufacturing Resource class represents a set of sub-classes - Manufacturing Automation Device, Communications Network, Material or Finished Part, Manufacturing Software, Equipment and Machinery, and Manufacturing Personnel. A Manufacturing Information Exchange class is further modeled as a set of sub-classes that facilitate the exchange of information structures between a collection of software objects that create, exchange, process, and store information items. These software objects are the sources and destinations of the information involved in the information flow with the rate and volume being gated by the number of transactions needed to support the material and control flows involved in a process.

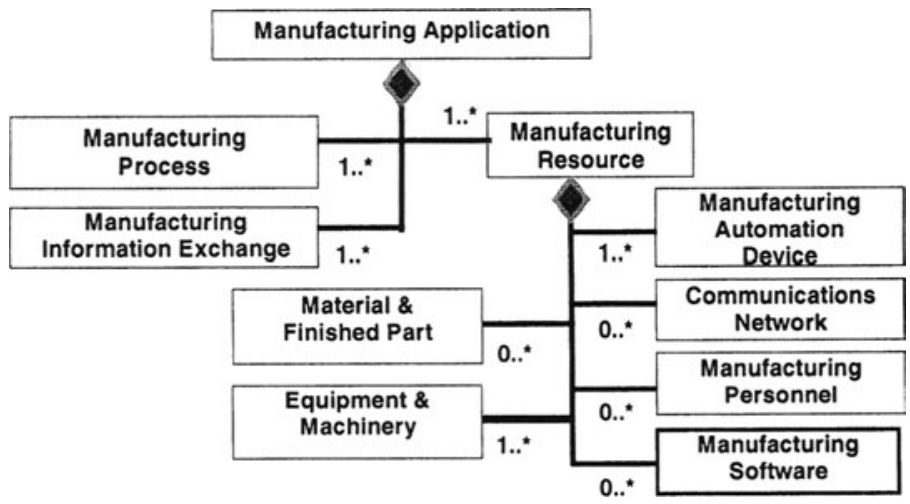

Figure 1: Integration Model View: Manufacturing Application

For example, in a material handling application, the timely delivery of a desired amount of a particular item to a certain destination, requires a set of "flow channels" that are interoperable. These "flow channels" may transport incoming raw material to a work-in-process area or finished goods from 
work-in-process to inventory or finished goods from inventory to shipping docks.

An integration model view is associated with each major class composing a Manufacturing Application class. A UML package consisting of a class diagram, a sequence diagram, and a deployment diagram represents each integration model view, per the ISO 15745, (2002) standard. A sequence diagram shows the series of transactions between the cooperating objects and the interfaces through which the object classes exchange material, information, or energy. These interfaces are further identified in the deployment diagrams and in the class diagrams. A set of integrated activities can only support the desired flow of materials, information, and other manufacturing resources, if the interfaces are interoperable, i.e. whatever is exchanged across the interface can be identified, recognized, understood, and properly handled by both resources collaborating via the interface.

\subsection{Integration models and interfaces}

The use of standard interfaces to elaborate an integration strategy is an obvious and proven approach. Integration of incompatible resources from different suppliers can be realized using interface adapters, converters, or gateways, but only to a lower degree of integration. A higher degree of integration can be achieved when the common interfaces are configured to interoperate in an optimal fashion, with very minimal use of gateway devices. In addition, use of interfaces based on open system standards - either formal international standards or specifications generated through industry consensus, will favor a broader source of suppliers.

A natural extension of such an approach is the use of an object-oriented integration model view that identifies the interfaces exposed by the component object classes of an application system model and then describes their compatible configurations in a systematic and web-ready form.

\subsubsection{Process integration and functional interoperability}

A manufacturing process consists of one or more activities, where each activity is associated with the performance of a specific function. Each function has to be performed in the right sequence, at the right time, at the right place, on the right target, by the right resource, with the expected outcome, in order to insure the desired flow of material, information, and energy. When the rate, volume, and quality of the process output meet the production goals, the activities of the manufacturing process are considered to be well integrated and the functions involved being interoperable. 
Using the ISO 15745, (2002) application integration model, the various types of process interfaces are identified, chosen and configured in a compatible manner to sustain the flow type and the flow rates per the requirements of an application. The attributes of each process interface type - input, output, setup, monitoring, can be defined using the generic definitions in ISO 18629, (2002) and the activity-specific definitions in IEC 62264, (2002). The inter-process exchanges and their synchronization can be described in terms of the generic and activity-specific attributes. The set of configurations of the selected process interfaces can be expressed as a set of XML files and the corresponding schemas. A set of XML-based configuration statements (XML, 2000/2001) can serve as a reference set of interoperable configurations for such types of application. This reference interoperability statement can then be used to select the resources and the information structures necessary to support the required flows between the processes.

\subsubsection{Resource Integration and performance interoperability}

Following the ISO 15745, (2002) framework, the resources that implement the processes enable the material flows by coordinating the activities and operations using associated information exchanges. Using the framework's resource integration model view, the various types of exposed resource interfaces are similarly identified, chosen, and configured to support the reference interoperability statement for process integration. Each resource, such as, a device, a machine, or a person, may have one or more types of interfaces - input, output, setup, monitoring, power, and environmental. The use of common standard interfaces, such as those promoted by IEC, ISO, or some industry organization, enables the resources to transfer materials and information with each other.

For example, a device's communication network interface may conform to specific clauses in the IEC 61158 , (2000) fieldbus standards, where the parameters in each clause are set to a range of values, as required by the application process. The values are chosen in order to support the bandwidth, response time, and coverage requirements of the application. These communication interface configurations, common to the inter-operating devices may be denoted in an XML file that is organized by its corresponding XML schema (XML, 2000/2001).

A set of XML files and related schema serves as a reference statement of interoperability for the group of resources used in a manufacturing application process. These statements of resource interoperability support the statement of process interoperability. A reference statement can be re-used in similar applications to verify if the proposed resources can coordinate and 
perform the functions needed to conduct the material and information flows at the desired rate, volume, quality, and cost.

\subsubsection{Information exchange integration and data interoperability}

In a similar manner, the ISO 15745 , (2002) integration framework defines an information exchange model view that exposes the software interfaces and the other information handling units that enable the flow of information required to control the manufacturing process. The information handling objects are distributed among the nodes on a communication network. The nodes are distributed among the devices, which in turn are distributed among the machines, equipment, materials, and operators.

A transaction between two objects occurs at the information exchange interfaces that have been configured to support the rate, volume, and response time of information exchanges. The different types of messages being sent from one object to another object are associated with a set of interfaces that define the services, protocol, and data structures. In general, the interfaces used to setup the messaging paths may be different from those used to convey the messages. The message origin, destination, size, structure, rate, latency, freshness, fidelity, security, and persistence determine the type of interface to be used. These information exchange interfaces are chosen to support the manufacturing process requirements.

For example, the OPC-DA, (2000) Data Access specification defines the generic interface services that may be used to access the data structures and their current values in a particular OPC server used in a material handling system. The meaning of the data structures and the specific formats can be the definitions specified in ISO 13374, (2002) for monitoring the machinery used in a process.

The particular settings for each software interface specification that is used in the application process to meet the information exchange requirements can be summarized in a set of XML files and related schema (XML, $2000 / 2001$ ), as recommended in ISO 16100, (2002). The set of XML files serves as reference statements of interoperability for the software components that exchange the data structures and handle the corresponding semantics. These statements of information exchange interoperability support the statements of process and resource interoperability.

\subsection{System integration and application interoperability}

Practical system integration within an application is realized when the processes, the resources, and the information exchanges involved are all served by interoperable interfaces. The combined sets of XML files and re- 
lated schema (XML, 2000/2001) that represent the various types of reference interoperability statements form an application interoperability statement or profile. The interoperability profile captures the application requirements in terms of the different types of interfaces and their specific configurations.

For example, in designing a material handling application, the selection and configuration of the interfaces for the material transport equipment, escort memory subsystem, and inventory control and management subsystem depend on several time-dependent and site-specific variables. Examples of these variables are the type of unit loads, number of orders issued, number of shipments, finished goods inventory, work-in-process inventory, throughput, average time to fill an order, and other production related parameters. Unless the material handling interfaces are configured to support the flows demanded by the production goals, the various subsystems could not transport the unit, batch, or streaming loads as needed by the application. Furthermore, if the information handling interfaces are not interoperable, then the information flows will not match the material flow and will result in loss of tracking and control.

\subsubsection{Uses of interoperability profile and integration models}

Interoperability is the ability of two or more systems or applications to exchange information and use the information that has been exchanged (note that the applications may be wholly resident on the same system or distributed across multiple systems).

The availability of a reference application interoperability profile enables an application developer to verify if a proposed resource implementation (i.e. a product) from a supplier provides all the required functional interfaces and whether these interfaces can be configured to operate within a specified performance range.

If each device, equipment, machine, or software being procured has a required interoperability profile, then matching the offered profiles with the reference profiles can facilitate the interoperability verification process.

The set of UML diagrams describing an integration model view - class, sequence, use case, state, and deployment, also provides reference descriptions of the application's detailed process behavior and resource attributes. These diagrams can be helpful in the design, operation, and maintenance of a manufacturing application.

\subsubsection{Safety, environmental, and security considerations}

Other application considerations can be included in both the interoperability profile and the integration models. For example, the reduction in the 
amount of scrap and waste, as well as, the efficient use fuel and energy, both contribute towards a more environmental-friendly design of the manufacturing process. The supporting interfaces and the associated criteria can be treated within the integration framework, as well. Product components that do not require special disposal procedures upon product obsolescence can be noted in an extended interoperability profile.

The safe and secure operation of a manufacturing process depends largely on the types of interfaces selected for the monitoring and control of those activities that pose a risk to the health and safety of plant floor personnel. A secure operating platform provides appropriate control over access to the critical information resources that run the system. Again, use of properly matched evaluation assessment levels of security to the known security targets can be verified as part of the interoperability checking process. The use of the Common Criteria specification in ISO/IEC 15408 (2000) can provide a consistent scheme for evaluating vulnerabilities and assessing appropriate security measures.

\section{INTEROPERABILITY SCENARIOS}

A key to the practical use of the framework approach described above is the availability of standard interfaces that can be tailored to inter-operate. The choices for standard interfaces are based on these interfaces being configured to exchange materials and information at the desired rate and volume. For instance, the method of denoting process interfaces in ISO 15745, (2002) and the method of describing a process in ISO 18629, (2002) must have a shared ontology for use in the manufacturing process domain. The shared ontology may be derived from the definitions in IEC 62264, (2002), Enterprise-Control System Integration. In this effort, the processes and their component activities are described relative to an enterprise model view, generically defined in ISO 15704, (2002). In IEC 62264, (2002), the process boundaries are chosen based on the types of information exchanged between the production control system and the other enterprise business functions.

An example of a set of areas that need alignment and harmonization among the various standards is given in Table 1 . The rows list the standards providing interface specifications while the columns list the standards providing integration and interoperability frameworks. Each table element identifies the set of definitions, in each standard, that have to be aligned in order for the two standards to be used consistently within the same application. To address different types of applications in various industry sectors will require a more comprehensive table involving other standards, showing the areas needing alignment and harmonization of definitions. Future standards can be 
included in these tables, indicating the needed interoperability with existing standards.

Table 1: Common Areas That Need to Be Aligned Between Standards

\begin{tabular}{|c|c|c|c|}
\hline & $\begin{array}{l}\text { ISO } 15704 \\
\text { Enterprise } \\
\text { Modeling }\end{array}$ & $\begin{array}{l}\text { ISO } 15745 \\
\text { Application Inte- } \\
\text { gration Frame- } \\
\text { work }\end{array}$ & $\begin{array}{l}\text { ISO } 16100 \\
\text { Manufacturing } \\
\text { Software Capa- } \\
\text { bility Profiling }\end{array}$ \\
\hline $\begin{array}{l}\text { IEC 62264: Enterprise- } \\
\text { Control System Integra- } \\
\text { tion }\end{array}$ & $\begin{array}{l}\text { Enterprise } \\
\text { model }\end{array}$ & $\begin{array}{l}\text { Integration mod- } \\
\text { els }\end{array}$ & $\begin{array}{l}\text { Capability } \\
\text { classes }\end{array}$ \\
\hline $\begin{array}{l}\text { ISO 18629: Process } \\
\text { Specification Language }\end{array}$ & $\begin{array}{l}\text { Process life- } \\
\text { cycles }\end{array}$ & $\begin{array}{l}\text { Process descrip- } \\
\text { tion }\end{array}$ & $\begin{array}{l}\text { Process descrip- } \\
\text { tion }\end{array}$ \\
\hline $\begin{array}{l}\text { ISO 13374: Condition } \\
\text { monitoring \& diagnostics } \\
\text { of machines }\end{array}$ & $\begin{array}{l}\text { Asset man- } \\
\text { agement } \\
\text { model views }\end{array}$ & $\begin{array}{l}\text { Information ex- } \\
\text { change and re- } \\
\text { source models }\end{array}$ & $\begin{array}{l}\text { Capability } \\
\text { model }\end{array}$ \\
\hline $\begin{array}{l}\text { ISO 20242: Application } \\
\text { Service Interface }\end{array}$ & $\begin{array}{l}\text { Enterprise } \\
\text { modeling }\end{array}$ & $\begin{array}{l}\text { Device interop- } \\
\text { erability profiling }\end{array}$ & \\
\hline $\begin{array}{l}\text { Pre EN ISO } 19439 \& \\
\text { 19440: Enterprise model- } \\
\text { ing }\end{array}$ & $\begin{array}{l}\text { Generic } \\
\text { model views }\end{array}$ & & \\
\hline $\begin{array}{l}\text { Device Profile Guideline } \\
\text { (IEC 65A - 65/290/DC) }\end{array}$ & & $\begin{array}{l}\text { Device integra- } \\
\text { tion model }\end{array}$ & \\
\hline $\begin{array}{l}\text { ISO 10303: Application } \\
\text { Protocols }\end{array}$ & $\begin{array}{l}\text { Enterprise } \\
\text { lifecycle } \\
\text { modeling }\end{array}$ & $\begin{array}{l}\text { Resource integra- } \\
\text { tion model }\end{array}$ & \\
\hline
\end{tabular}

There are additional considerations for harmonized definitions among the row elements. For example, activity descriptions and data models in IEC 62264, (2002) and ISO 13374, (2002); data definitions \& structures in IEC 62264, (2002) and pre EN ISO 19440, (2002); process descriptions in ISO 18629, (2002) and ISO 10303, (1999); resource views in ISO 15745, (2002) and ISO 15704, (2001); function views in ISO 16100, (2002) and ISO 15704, (2001); profile templates in ISO 16100, (2002) and ISO 15745, (2002).

The IEC 62264, (2002) standard also provides definitions of data units exchanged between a subset of functional activities that comprise the various enterprise processes. These activities are organized and performed in a particular sequence when a specific product is taken through its lifecycle planning, design, implementation, production, distribution, and disposition. By correlating the activity definitions specified in the ISO 10303, (1999) STEP application protocols (AP) with the activity definitions defined in IEC 62264 , (2002), the associated data structures being exchanged can be compared with respect to syntax, semantics, and synchronization. 
Use of an application integration framework facilitates the construction of a set of manufacturing application system models and the compilation of the standard interfaces, identified within the models, as required by the system resources that enable the manufacturing processes.

Using the combined integration model views for the process, resource, and information exchanges, a list of interfaces required to support the flows can be identified. The desired or as-built interface configurations can be described in a set of XML files, using the XML schemas defined in the ISO 15745, (2002) application integration framework standard. For each configured interface, an XML file contains the particular settings or values assigned to the interface parameters. The suite of XML files and the corresponding schemas can be labeled as the interoperability profile for a particular manufacturing application. The specific rules used to select the interface parameter values, based on the application's needs, can also be documented in a complementary set of XML files and schemas (XML, 2000/2001). The conditions applied to the integration model views can be described in terms of constraint statements attached to the UML diagrams for the various integration model views.

The models and the associated interfaces capture the control and coordination mechanisms needed to support the interoperability of the set of processes within the application system. The parameters and options detailed in the set of standard specifications associated with the required interfaces can be selected and verified to provide a set of compatible configurations. These compatible configurations of interfaces can represent a system solution that meets the requirements of a manufacturing application system. Similar manufacturing applications within an enterprise, a supply chain, or an industry can use a set of compatible interface configurations as a starting basis for developing more specific solutions that support system interoperability.

The statement of the interoperability of a set of standards used in an application can only be realized if the standards have been harmonized. A more comprehensive table of interoperable standards, similar to the chart in Section 3, needs to be developed. The elements in the charts have to be harmonized to support application integration. The critical effort for standards committees is to collaborate and to coordinate their results to achieve the interoperability of the standards, plus targeted industry dialogues. More efficient methods are needed to enable the collaboration and coordination, including web-based meetings, common repositories, and joint efforts. 


\section{REFERENCES}

IEC 61 158, (2000), Industrial Process Control and Measurement Systems - Digital Communications: Fieldbus.

IEC 61512, (1997), Batch Control.

IEC 62264/FDIS, (2002), Enterprise-Control System Integration.

IEC 65A-65/290/DC, (2002), Device Profile Guideline.

ISO 10303, (1999), Product data representation and exchange.

ISO 13374/FDIS, (2002), Condition monitoring and diagnostics of machines - Data processing, communications and presentation.

ISO 15704, (2001), Requirements for Enterprise Reference Architectures and Methodologies. ISO 15745/FDIS, (2002), Industrial automation systems and integration - Open systems application integration framework.

ISO 16100/FDIS, (2002), Industrial automation systems and integration - Manufacturing software capability profiling for interoperability.

ISO 18629/DIS, (2002), Industrial automation system and integration - Process specification language.

ISO 20242, (WD/2002), Industrial automation systems and integration -Application Service Interface.

Pr EN ISO 19439, (2002), Enterprise Integration - Framework for Enterprise Modelling.

Pr EN ISO 19440, (2002), Language Constructs for Enterprise Modelling.

ISO/IEC 15408, (2000), Information technology-Security techniques -Evaluation criteria for IT security.

ISO/IEC 19501, (2001), Information technology-Unified Modeling Language (UML).

OPC DA, (2000), Open Process Control: Data Access Specifications.

XML, REC-xml-20001006, (2000), Extensible Markup Language (XML) 1.0 Second Edition - W3C Recommendation 6 October.

XML, REC-xmlschema-1/2-20010502, (2001), XML Schema Part 1/2: Structures/ Data types - W3C Recommendation 02 May. 